\title{
Mechanistic studies on
} cationization in MALDI-MS

employing a split sample plate set-

up

\section{Journal Article}

Author(s):

Metternich, Jonas B.; Czar, Martin F.; Mirabelli, Mario F.; Bartolomeo, Giovanni L.; Zouboulis, Konstantin C.M.; Zenobi, Renato 1

Publication date:

2019

Permanent link:

https://doi.org/10.3929/ethz-b-000366326

Rights / license:

$\underline{\text { In Copyright - Non-Commercial Use Permitted }}$

Originally published in:

Journal of the American Society for Mass Spectrometry 30(11), https://doi.org/10.1007/s13361-019-02291-7 


\title{
Mechanistic studies on cationization in MALDI- MS employing a split sample plate set-up
}

\author{
Jonas B. Metternich, Martin F. Czar, Mario F. Mirabelli, Giovanni L. Bartolomeo, \\ Konstantin C. M. Zouboulis, Renato Zenobi* \\ ETH Zurich, Department of Chemistry and Applied Biosciences, $\mathrm{CH}-8093$, Zurich, Switzerland
}

Email zenobi@org.chem.ethz.ch

\begin{abstract}
In the analysis of polymers by matrix-assisted laser desorption/ionization mass spectrometry (MALDI-MS), a commonly observed ionization pathway is cation adduct formation, as polymers often lack easily ionizable (basic/acidic) functional groups. The mechanism of this process has been hypothesized to involve gas-phase cation attachment. In previous experiments, a split sample plate set up has been introduced, enabling separate deposition of the components on individual MALDI plates. The plates are divided by a small gap of a few micrometers, allowing simultaneous laser irradiation from both plates, while precluding the possibility of any other interactions prior to ablation. Here, we extend on these studies by using different polymer-salt combinations to test the generalizability of a gas phase ionization process. Clear evidence for in-plume ionization is presented for the model polymers poly(methyl methacrylate) and polystyrene. Furthermore, the contribution of in-plume processes to the overall ion formation by cationization is considered, providing a first estimate for the importance of this pathway.
\end{abstract}

\section{Introduction}

Synthetic polymers are an important class of analytes accessible by matrix assisted laser desorption ionization mass spectrometry.[1-4] Since polymers often lack basic or acidic functional groups, the predominant ionization pathway in MALDI-MS is cationadduct formation.[5] Ionization via cation-adduct formation is governed by the dynamics of both desorption and charge separation. However, neither the timescales of these processes, nor the sequence in which they occur, are fully understood. The two main pathways commonly considered to contribute to cationization are a preformation of cation-adducts in solution [6-9] and an in-plume cation attachment[7, 10, 11]. In the former case, cation-adducts are proposed to be formed in solution, during sample preparation. Upon deposition the adduct co-crystallizes with the respective counterion. Laser irradiation provides the energy required for counterion separation and release of the adduct. [8, 12] In contrast, within the in-plume hypothesis, it is assumed that the adducts are not preformed in the crystal. Instead, the laser irradiation generates free cations and polymer molecules, which can form adducts upon collision within the dense MALDI plume.[7]

Several studies have been presented in favor either mechanism. Lehmann et al.[6, 9] showed that some strongly binding complexes exhibit a striking correlation between their mass spectral patterns and their solution- phase complexation behaviour. The feasibility of inplume cationization, on the other hand, has been addressed by a variety of experimental approaches, for example a layered deposition $[6,13,14]$ and solvent free sample preparation $[10,11]$ to avoid preformation. The most convincing pieces of evidence were given by Belov et al. [15] and Erb et al. [16] Both studies employed a special experimental set-up, which utilizes separate target plates for polymer and cation generation. The physical separation excludes preformation, while in-plume processes are feasible by mixing of the plumes. The split sample plate developed by Erb et al.[16] is especially convincing as the material is ablated by a single laser shot from two plates separated only by a gap of a few micrometers. These results clearly show that in-plume ionization is possible for some analytes. It is, however, still unclear if this also applies to different polymer classes and to which degree other ionization pathways might contribute. Jaskolla and Karas[17] demonstrated that protonated species can be formed either by preformation or by inplume proton transfer, which highlights the question whether cationization occurs similarly.

Here, we present additional studies performed on a split plate set-up, similar to the one employed by Erb et al.[16] By investigation of different polymers and cationizing agents, we generalize earlier findings and adress questions regarding the overal contribution and importance of in-plume processes for cationization in 
MALDI. A first estimate for the relative contribution of in-plume ionization is provided.

\section{Experimental}

Material

Size exclusion chromatography (SEC) standards of Polystyrene 2000 (PS, Mw= $1890 \mathrm{Da}, \mathrm{Mn}=1790 \mathrm{Da}$, $\mathrm{Mp}=1920 \mathrm{Da}, \mathrm{PDI}=1.06$ ) and poly (methyl methacrylate) 2000 (PMMA, Mw= $2130 \mathrm{Da}, \mathrm{Mn}=1920 \mathrm{Da}, \mathrm{Mp}=2160$ $\mathrm{Da}, \mathrm{PDI}=1.11$ ) were obtained from Sigma Aldrich (St. Louis, MO, USA). Mass distributions were determined by the manufacturer using size exclusion chromatography.

Furthermore, trans-2-[3-(4-tert-Butylphenyl)-2methyl-2-propenylidene]malononitrile (DCTB, $\geq 98 \%$ ), copper trifluoroacetate, silver trifluoroacetate $(99.99+\%)$ and ethylendiamine diacetate (EDDA, 99\%) were acquired from Sigma Aldrich. Lithium trifluoroacetate (97\%) was purchased from Alfa Aesar (Ward Hill, MA, USA). Tetrahydrofuran, methanol and water were obtained in HPLC grade quality from various vendors. All chemicals were used without further purification. Samples were prepared by pneumatic spray deposition of solutions containing matrix and either polymer or salt. A description of the spraying setup and procedure is given in the supplementary information.

\section{Instrumentation}

Measurements were performed on an Autoflex I MALDI-ToF mass spectrometer (Bruker Daltonics $\mathrm{GmbH}$, Bremen, Germany). The sample plate has been described elsewhere.[18] Briefly, cavities were milled into a commercial MTP 384 target plate (Bruker Daltonics) to hold one set of five small target plates (see Figure SI-1). The target plates are coated separately and joined prior to the measurements. Screws are used to secure the plates and avoid displacement during analysis.

Unless stated elsewise, the same plates were used for all experiments. The gap width between two plates was determined to be $5 \pm 2 \mu \mathrm{m}$ and $14 \pm 2 \mu \mathrm{m}$ without and with the addition of a steel foil (10 $\mu \mathrm{m}$ thickness) spacer, respectively. A spacer was used in all experiments unless otherwise stated, to prevent any transfer across the gap. Each foil was only used for a single experiment. The ablation profile for the setup was found to be elliptical (111 $\mu \mathrm{m}$ and $57 \mu \mathrm{m}$ semi-axes). A laser fluence of $0.19 \mathrm{~J} / \mathrm{cm}^{2}$ and an extraction delay time of $280 \mathrm{~ns}$ were employed in all experiments, including a constant internal instrumental delay of approximately $80 \mathrm{~ns}$, which was determined previously.[18]

Due to the low intensities observed at the junction, a high detector bias voltage was required to obtain sufficient spectral quality. We note that, as a result of these settings, the most abundant matrix signals led to detector saturation. However, this did not have any impact on the formation of cation adducts. This was confirmed by control experiments on a plate containing only salt or polymer, which did not exhibit the adduct signal, despite detector saturation (Figure SI-8 and SI9).

\section{Results and Discussion}

Polystyrene (PS) and poly(methyl methacrylate) (PMMA) were selected as representatives of different polymer classes. PS was chosen as a model compound for non-polar polymers containing unsaturated hydrocarbon chains or aromatic systems. Binding to transition metal cations, such as copper or silver, is facilitated by $d-\pi$ orbital interactions involving the aromatic ring of the polymer.[5] PMMA was chosen as a second model compound to reflect the binding of alkali cations to polar functional groups of polymers, which is dominated by ion-dipole interactions.[19] As cationizing agents, we chose copper and silver for polystyrene and lithium for poly(methyl methacrylate), due the high ionization efficiency of these combinations.[11, 20] Trifluoroacetate salts were chosen, because of their solubility in organic solvents, making it compatible with the hydrophobic matrix and polymer.

\section{In-plume cation-adduct formation}

Split-plate MALDI experiments (see Figure 1) were performed to assess whether in-plume processes are involved in the formation of polymer-cation adducts. In a split-plate experiment, one plate (hereafter denoted as the "salt plate") was coated with cationizing agent (deposited as its trifluoroacetate salt) and DCTB matrix, whereas the other plate (denoted as the "polymer plate") was coated with polymer and DCTB matrix. Figure 1 a-c compares MALDI mass spectra for different polymer-cationizing agent pairs measured by ablation of the polymer plate, far from (gray traces) and in close proximity to (black traces) the junction. Spectra measured by ablation of only the polymer plate show no discernible signals corresponding to the ionized polymer; likewise, no signals corresponding to polymer ions are seen when ablating only the salt plate, as expected (see Figure SI-10). In stark contrast, polymer-cation adducts are clearly visible upon ablation of the region near the junction. This result strongly suggests that ionization requires the simultaneous release of polymer and cationizing agent into the MALDI plume.

To qualify this further, the integrated signal intensity of the most abundant polymer signals was monitored when approaching the junction in small increments (see Figure SI-6 a). A step size of $25 \mu \mathrm{m}$ was chosen, which 
is smaller than the laser beam diameter $(111 \mu \mathrm{m}$ diameter perpendicular to the junction axis). Each ablation position was offset by $150 \mu \mathrm{m}$ along the junction axis to avoid overlapping spots. Representative results of this diagonal scan across the junction are shown in Figure 1. In every experiment, a distinct maximum in the integrated polymer-cation adduct signal is observed within $50 \mu \mathrm{m}$ of the junction. Signals decrease rapidly with increasing displacement from this position and disappear within $50 \mu \mathrm{m}$. Adduct signals are thus observed for only one or two, and rarely three, spots for each experiment. This result is in agreement with the theoretical maximum of four overlapping ablation positions resulting from a gap size of $14 \mu \mathrm{m}$ and a laser beam diameter of $111 \mu \mathrm{m}$ perpendicular to the junction axis.

Each diagonal scan shown in Figure 2 was replicated several times to ensure reproducibility of the results (see Figure SI-7). We found that, although each scan shows identitical qualitative behavior, there is some variability in the ablation position that gives maximum signal intensity. Namely, across all experiments, the nominal position of maximum intensity occurs at either $0 \mu \mathrm{m}$ or $+25 \mu \mathrm{m}$. We attribute this slight systematic shift of the maximum intensity towards the polymer plate to a misalignment between the intended and actual ablation positions. This seems reasonable, given the limited resolution of the camera used for alignment, and the fact that initial positioning was done by manual alignment, which was necessary since the plate and the motor axes are slightly mismatched for this instrument. However, it should be noted that contributions from differences in ablation efficiencies for the polymer versus cationizing agent cannot be ruled out.

Finally, to exclude carry-over effects from previous experiments as a reason for the observed shift, additional control experiments were performed, in which only a single target plate was coated. Therefore, only polymer or cationizing agent were present at the junction and successful cation-adduct formation would have indicated carry-over of the missing reactant.This was, however, not observed in either case (see Figure SI-8 and SI-9). As the results presented here were obtained by summation of twenty laser shots per ablation position, additional control experiments were required to assess the possibility of redeposition of neutral particles released from the MALDI plume between ablation events. To assess this possibility, single-shot experiments were performed at the junction, thus excluding redeposition. For all polymer/cation pairs examined, polymer-cation adduct signals are clearly present in the single-shot experiments (see Figures SI-10). This finding is in agreement with the work performed by Erb et al.[16] and suggests that particle redeposition is not the sole origin of ionization in the experiments presented above. Contributions can, however, not be excluded, if several laser shots are being acquired for each spot.
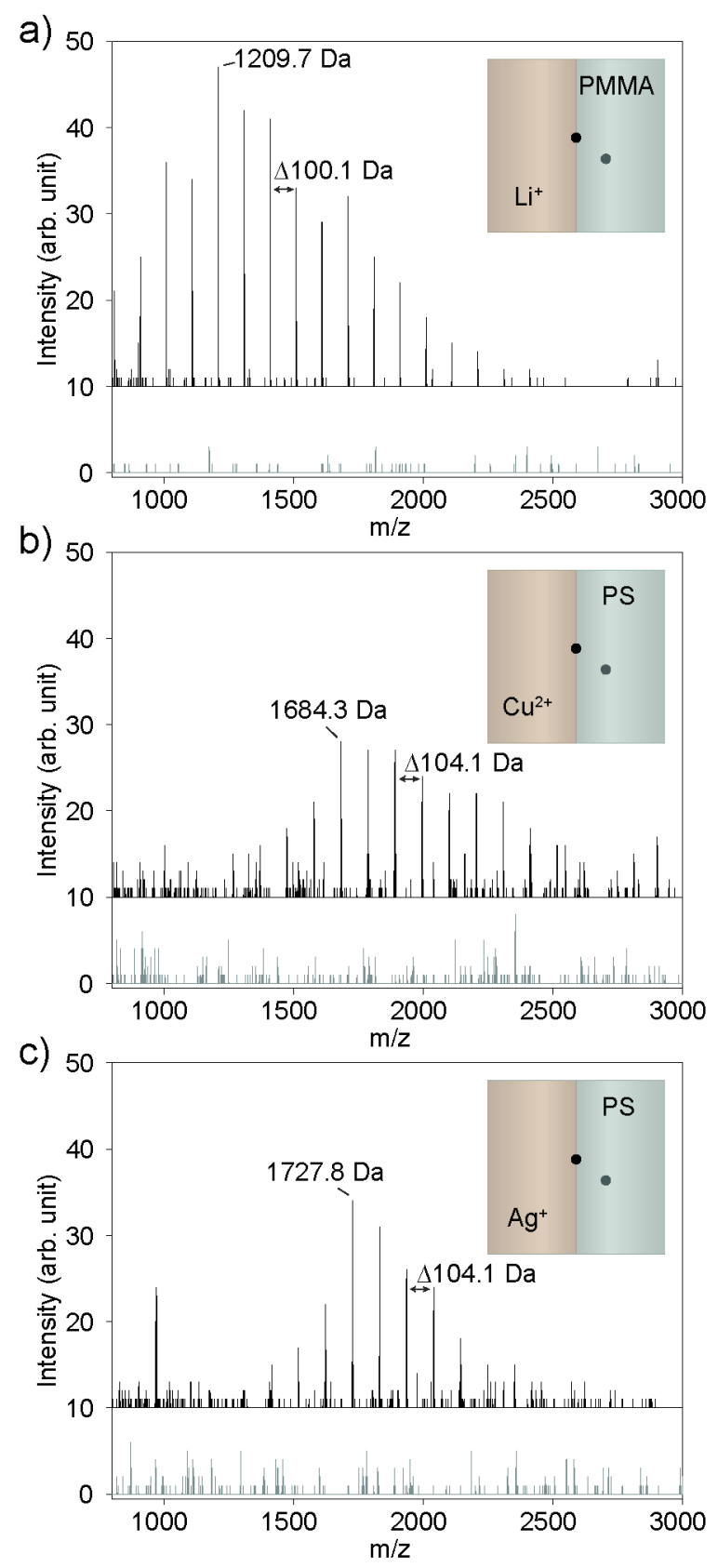

Figure 1: MALDI mass spectra (a-c) from split-plate experiments using PMMA-Li (a), PS-Cu (b) and PS-Ag (c). A schematic representation of the split plate and two ablation positions is given in the top right corner. The black traces are the mass spectra acquired near the junction, whereas the grey traces are those measured by ablating the polymer plate only (sum of 20 shots in both cases). The black traces are offset vertically by 10 counts for ease of comparison. The peaks correspond to singlycharged polymer-cation adducts. 


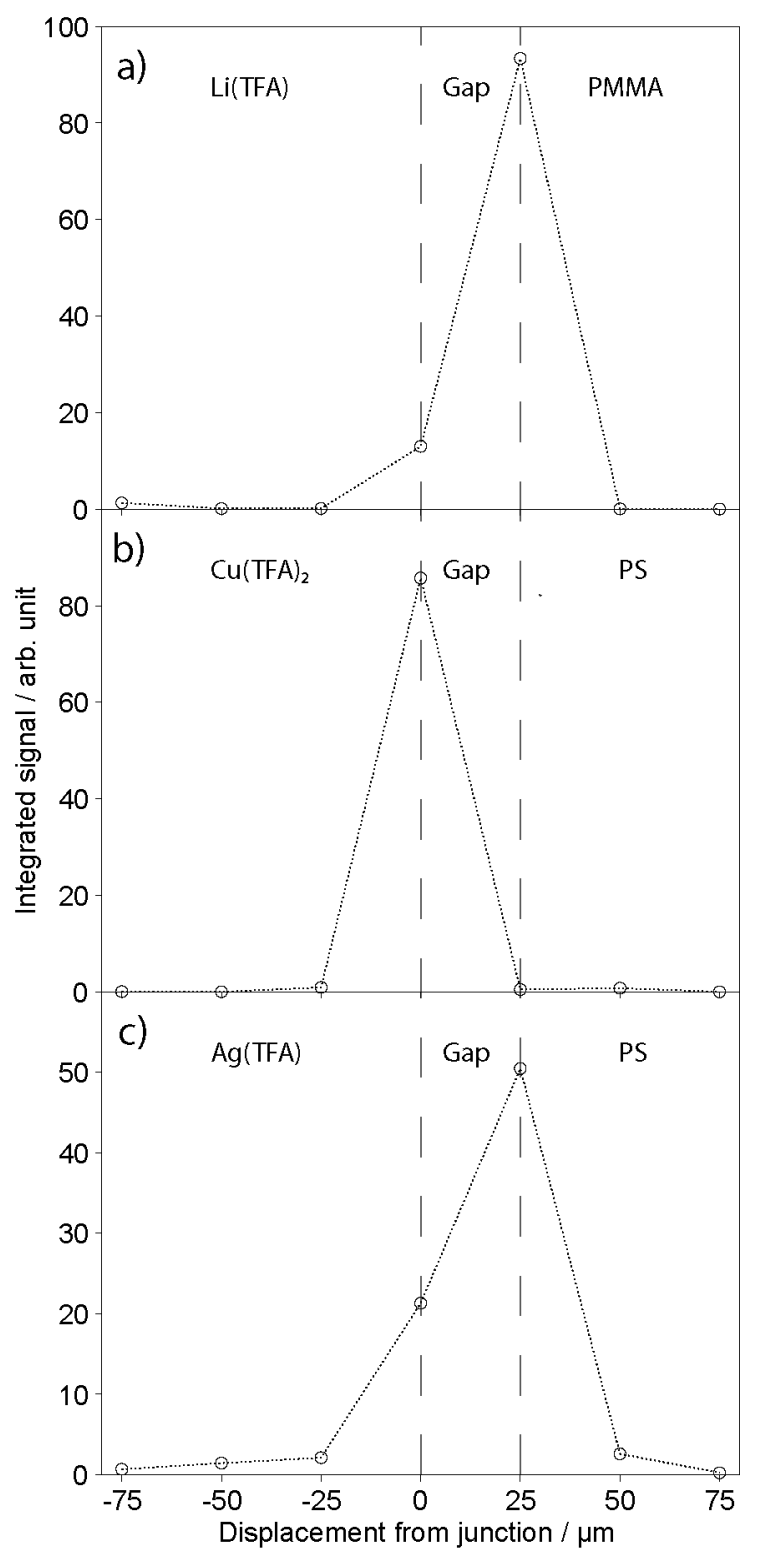

Figure 2: Integrated signal intensities PMMA-Li (a), PS$\mathrm{Cu}$ (b) and PS-Ag (c) adducts as a function of displacement from the junction. Negative values correspond to the plate coated with matrix and metal salt, whereas positive values represent the plate containing polymer and matrix. The estimated positions of the plates and gap are indicated by dashed lines.

\section{Contribution of in-plume processes to cationization}

The results presented above provide strong qualitative evidence that in-plume processes take place. However, the degree to which in-plume processes contribute to the overall polymer-cation signals observed remains unclear. As MALDI measurements on polymers are commonly performed by co-deposition, it is still possible that the majority of ions are formed by alternate pathways, most importantly a preformation. To obtain an estimate of the contribution of in-plume cationization, we carried out a comparison of co-deposited and separately deposited (split plate experiment) polymer and salt samples. Codeposited samples were prepared on a single MALDI plate, unlike the separate deposition, which required two plates. Thus, the concentrations used for the codeposited samples were half of those used for the splitplate samples. This was to ensure that the total amount of polymer and cationizing agent deposited relative to the ablated surface area was kept roughly the same for the two experiments. Figure $3 a$ compares a MALDI mass spectrum obtained by ablation of co-deposited PMMA and LiTFA on a single MALDI plate (black trace), to one using the joint plate with separately deposited polymer and cationizing agent (grey trace), as described above. Notably, the spectrum obtained by the split plate experiment exhibits a clear shift towards lower masses relative to the spectrum measured for a co-deposited sample. Since the split plate increases the distance between the reactants, a mass discrimination based on their velocity may result. Additionally, it must be considered that the distribution of oligomers within the plume might not be homogeneous. In either case, a shange of the mass distribution towards higher masses would be expected, if the distance is decreased by the removal of the stainless steel spacer.

However, this was not observed experimentally (see Figure SI-12). Similarly, no effect of the laser fluence on the mass distribution was found (see Figure SI-14). Therefore, the shift in the apparent mass distribution might indicate a preferential ionization of large oligomers by pathways other than in-plume cationization. This would be in agreement with the findings of Jaskolla and Karas[17], who reported a higher contribution of preformation for protonation of larger molecules, whereas in-plume processes dominated for small molecules. We note, however, that a sample preparation related origin of the mass shift cannot be excluded. Sample preparation has been described previously[21, 22] as an important factor in the determination of mass distributions. To ensure the ablation of equal amounts of each substance for codeposition and split-plate experiments, different matrixto-analyte ratios were required. Therefore, the sample preparation was slightly different in terms of concentrations and solvents involved (see SI). Especially the lower matrix-to-analyte ratios could explain the observation, as they have been reported to favor smaller oligomers.[21] Additional split-plate experiments were performed, employing matrix-toanalyte ratios identical to those used in co-deposition experiments. No significant change in the mass distribution was observed towards higher masses (see Figure SI-13). 
a)

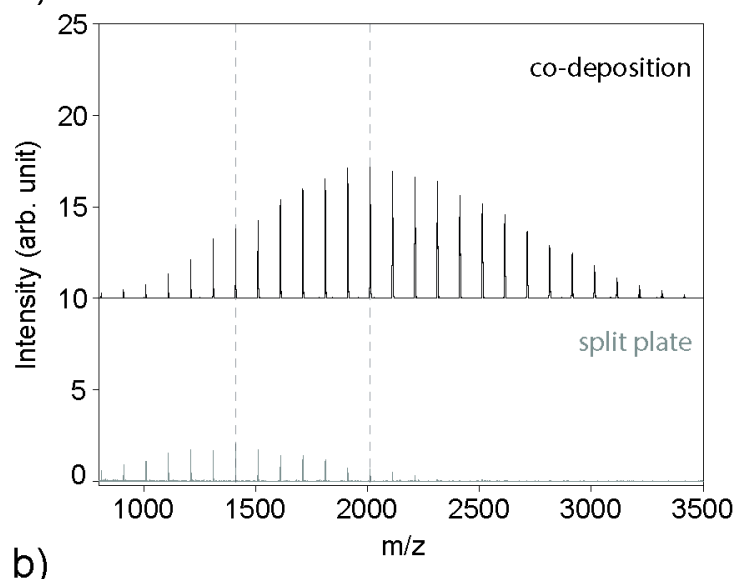

b)

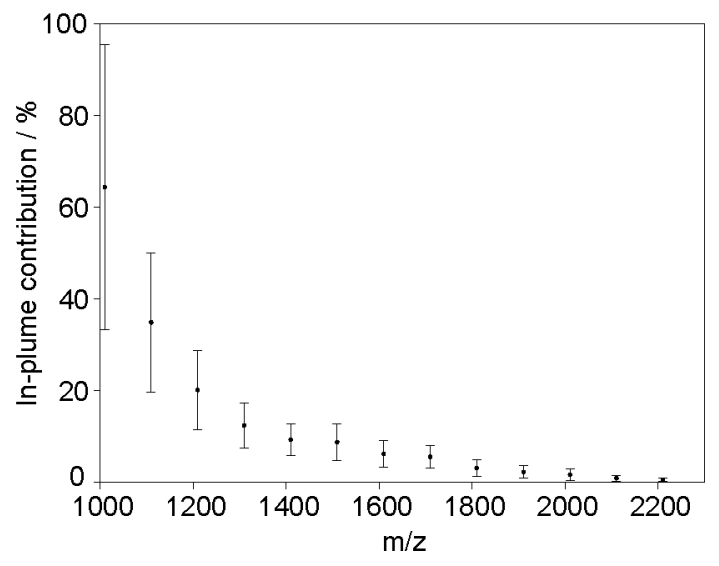

Figure 3: MALDI mass spectra (a) obtained by codeposition (black trace) and split plate experiments (gray trace), using PMMA and Li(TFA). Both spectra represent an average of fifty single shot spectra obtained on a single plate and at the junction, respectively. The black trace is offset vertically by 10 counts for ease of comparison. Relative contributions of in-plume cationization (b) were calculated for each PMMA-Li adduct individually, by division of the integrated signal intensities.

Figure $3 \mathrm{~b}$ compares the integrated peak intensities for each of the individual polymeric species shown in the mass spectra in Figure 3a. The data are shown as the ratio of the absolute abundance measured in the split plate experiment relative to that measured in the co-deposition experiments, using identical instrument settings. The lower mass PMMA-Li adducts $(<1000$ $\mathrm{Da}$ ) were not considered in the analysis due to their low abundance. These data can be considered as a relative measure of the contribution of in-plume cationization to the total intensity observed in the mass spectrum. However, due to the different mass distributions, contributions were calculated for each species. The relative contribution ranges from $64 \%$ to $9 \%$ for polymers containing ten to fourteen methylmethacylate subunits. We emphasize that these values should be only taken as lower bounds. This is due to the fact that a significant fraction of the ablated material, statistically at least half, will have insufficient radial velocity to merge with the other MALDI plume. Moreover, the split plate set-up includes a gap between the two MALDI plates, which reduces the total amount of material ablated. In this light, contributions are significant for low mass oligomers, whereas the picture for larger oligomers is less clear due to the unknown origin of the change in mass distribution.

\section{Conclusions}

The formation of cation adducts was monitored in the mixed plume at the junction between two MALDI plates, coated with either polymer or cationizing agent. Adducts were exclusively formed upon laser irradiation at or in close proximity to the junction. This demonstrates that polymer-cation adducts, exhibiting different kinds of electrostatic or complexing interactions, can be ionized by an in-plume process. A comparison of the signals obtained on the split plate set-up with those of a co-deposition experiment reveals a significant contribution of in-plume processes to the ionization of low mass oligomers. However, the relative contribution was found to decrease with increasing polymer mass. This could be due to a prefered ionization by alternate pathways, for example ion preformation, for larger oligomers. Although, a shift in the mass distribution due to the sample preparation conditions cannot be fully excluded either.

\section{Acknowledgment}

We would like to express our deep gratitude to the Fonds der chemischen Industrie for financially supporting our research (Kekulé Fellowship to Jonas B. Metternich). Further more, the authors would like to thank Dr. Adrien Marchand and Mr. Prince Tiwari for helpful discussion. They also thank Mr. Cristian Marro, Mr. Christoph Bärtschi and Mr. Heinz Benz for their outstanding technical support.

\section{Supplementary Material}

An electronic supplementary information is available online. Data used in this publication will be made freely accessible in a curated data archive at ETH Zurich (https://www.research-collection.ethz.ch) under the DOI 10.3929/ethz-b-000326850.

\section{References}

1 Drzeżdżon, J., Jacewicz, D., Sielicka, A., Chmurzyński, L.: MALDI-MS for polymer characterization - Recent developments and 
future prospects. TrAC Trends in Analytical Chemistry. 115, 121-128 (2019)

2. Nielen, M.W.F.: Maldi time-of-flight mass spectrometry of synthetic polymers. Mass Spectrometry Reviews. 18, 309-344 (1999)

3. Charles, L.: MALDI of synthetic polymers with labile end-groups. Mass Spectrometry Reviews. 33, 523-543 (2014)

4. Räder, H., Schrepp, W.: MALDI-TOF mass spectrometry in the analysis of synthetic polymers. Acta Polym. 49, 272-293 (1998)

5. Knochenmuss, R.: Ion formation mechanisms in UV-MALDI. Analyst. 131, 966-986 (2006)

6. Edda, L., Richard, K., Renato, Z.: Ionization mechanisms in matrix-assisted laser desorption/ionization mass spectrometry: contribution of pre-formed ions. Rapid Commun. Mass Spectrom. 11, 1483-1492 (1997)

7. Richard, K., Edda, L., Renato, Z.: Polymer Cationization in Matrix-Assisted Laser Desorption/Ionization. Eur. Mass Spectrom. 4, 421-427 (1998)

8. Karas, M., Glückmann, M., Schäfer, J.: Ionization in matrix-assisted laser desorption/ionization: singly charged molecular ions are the lucky survivors. Journal of Mass Spectrometry. 35, 1-12 (2000)

9. Lehmann, E., Zenobi, R., Vetter, S.: Matrixassisted laser desorption/ionization mass spectra reflect solution-phase zinc finger peptide complexation. Journal of the American Society for Mass Spectrometry. 10, 27-34 (1999)

10. Trimpin, S., Rouhanipour, A., Az, R., Räder, H.J., Müllen, K.: New aspects in matrixassisted laser desorption/ionization time-offlight mass spectrometry: a universal solventfree sample preparation. Rapid Commun. Mass Spectrom. 15, 1364-1373 (2001)

11. Hortal, A.R., Hurtado, P., Martínez-Haya, B., Arregui, A., Bañares, L.: Solvent-Free MALDI Investigation of the Cationization of Linear Polyethers with Alkali Metals. The Journal of Physical Chemistry B. 112, 8530-8535 (2008)

12. Karas, M., Krüger, R.: Ion Formation in MALDI: The Cluster Ionization Mechanism. Chemical Reviews. 103, 427-440 (2003)

13. Anne-Mette, H., David, M.H., Peter, J.D., James, H.S.: Letter: Evidence for Cationization of Polymers in the Gas Phase during Matrix-Assisted Laser Desorption/lonization. Eur. Mass Spectrom. 3, 471-473 (1997)

14. Hanton, S.D., Owens, K.G., Chavez-Eng, C., Hoberg, A.-M., Derrick, P.J.: Updating Evidence for Cationization of Polymers in the Gas Phase during Matrix-Assisted Laser
Desorption/Ionization. European Journal of Mass Spectrometry. 11, 23-29 (2005)

15. Belov, M.E., Myatt, C.P., Derrick, P.J.: Chemical ionization of neutral peptides produced by matrix-assisted laser desorption. Chemical Physics Letters. 284, 412-418 (1998)

16. Erb, W.J., Hanton, S.D., Owens, K.G.: A study of gas-phase cationization in matrix-assisted laser desorption/ionization time-of-flight mass spectrometry. Rapid Commun. Mass Spectrom. 20, 2165-2169 (2006)

17. Jaskolla, T.W., Karas, M.: Compelling Evidence for Lucky Survivor and Gas Phase Protonation: The Unified MALDI Analyte Protonation Mechanism. Journal of The American Society for Mass Spectrometry. 22, 976-988 (2011)

18. Mirabelli, M.F., Zenobi, R.: Observing Proton Transfer Reactions Inside the MALDI Plume: Experimental and Theoretical Insight into MALDI Gas-Phase Reactions. Journal of The American Society for Mass Spectrometry. 28, 1676-1686 (2017)

19. Smith, S.F., Chandrasekhar, J., Jorgensen, W.L.: Ab initio study of acid-base interactions. Proton, lithium, and sodium affinities of firstand second-row bases. The Journal of Physical Chemistry. 86, 3308-3318 (1982)

20. Zeegers, G.P., Steinhoff, R.F., Weidner, S.M., Zenobi, R.: Evidence for laser-induced redox reactions in matrix-assisted laser desorption/ionization between cationizing agents and target plate material: a study with polystyrene and trifluoroacetate salts. International Journal of Mass Spectrometry. 416, 80-89 (2017)

21. Schriemer, D.C., Li, L.: Mass Discrimination in the Analysis of Polydisperse Polymers by MALDI Time-of-Flight Mass Spectrometry. 1. Sample Preparation and Desorption/lonization Issues. Analytical Chemistry. 69, 4169-4175 (1997)

22. Dogruel, D., Nelson, R.W., Williams, P.: The Effects of Matrix $\mathrm{pH}$ and Cation Availability on the Matrix-assisted Laser

Desorption/Ionization Mass Spectrometry of Poly(methyl methacrylate). Rapid Commun. Mass Spectrom. 10, 801-804 (1996) 